\title{
Identification of Energy Metabolism Changes in Diabetic Cardiomyopathy Rats Using a Metabonomic Approach
}

\author{
Liangcai Zhao $^{\mathrm{a}}$ Minjian Dong $^{\mathrm{a}}$ Cuicui Xu $\mathrm{u}^{\mathrm{a}}$ Hong Zheng ${ }^{\mathrm{a}} \quad$ Tingting Wei $^{\mathrm{a}}$ \\ Kun Liu ${ }^{b}$ Zhihan Yan ${ }^{b}$ Hongchang Gao \\ aSchool of Pharmaceutical Sciences, Wenzhou Medical University, Wenzhou, Zhejiang, 'Radiology \\ Department of the Second Affiliated Hospital, Wenzhou Medical University, Wenzhou, China
}

\section{Key Words}

Diabetic cardiomyopathy $•$ Nuclear magnetic resonance $\cdot$ ATP $•$ Metabonomics

\begin{abstract}
Background/Aims: Diabetic cardiomyopathy (DCM) is a serious complication of diabetes. It is therefore crucial to elucidate the characteristic metabolic changes that occur during the development of diabetes to gain an understanding the pathogenesis of this disease and identify potential drug targets involved. Methods: ${ }^{1} \mathrm{H}$ nuclear magnetic resonance-based metabonomics combined with HPLC measurements were used to determine the metabolic changes in isolated cardiac tissues after 5 weeks, 9 weeks, and 15 weeks in rats treated with streptozotocin. Results: Pattern recognition analysis clearly discriminated the diabetic rats from time-matched control rats, suggesting that the metabolic profile of the diabetic group was markedly different from that of the controls. Quantitative analysis showed that the levels of energy metabolites, such as the high-energy phosphate pool (ATP and creatine), significantly decreased in a time-dependent manner. Correlation analysis revealed the inhibition of glycolysis and the tricarboxylic acid (TCA) cycle, enhanced lipid metabolism, and changes in some amino acids, which may have led to the decline in energy production in the DCM rats. Conclusions: The results indicated that the administration of energy substances or the manipulation of myocardial energy synthesis induced by increased glucose oxidation may contribute to the amelioration of cardiac dysfunction in diabetes.
\end{abstract}

\section{Introduction}

Diabetes mellitus (DM) is the most common metabolic disease in the world and a major public health concern. Changes in glucose metabolism are known to facilitate profound disturbances in the metabolism of lipids, amino acids, and energy, and has overwhelming and long-lasting injurious effects on different tissues and organs [1]. Among the complications 


\section{Cellular Physiology Cell Physiol Biochem 2018;48:934-946 \begin{tabular}{l|l} 
DOI: 10.1159/000491960 & Ond 2018 The Author(s). Published by S. Karger AG, Basel \\
www.karger.com/cpb
\end{tabular} \\ Zhao et al.: Metabolic Alterations in Diabetic Hearts}

of type 1 DM (T1DM) and type 2 DM (T2DM), diabetic cardiomyopathy (DCM) is lifethreatening complication due to impaired cardiac diastolic and systolic functions [2]. DCM is characterized by apoptosis, fibrosis, diastolic dysfunction, cardiomyocyte hypertrophy, and increased susceptibility to ischemic injury $[3,4]$. However, the pathogenic mechanisms underlying the disease are still far from being understood.

Recent evidence suggests that changes in cardiac energy occurs early in the pathophysiology of diabetes [5]. Furthermore, a characteristic of cardiomyopathies in hyperglycemia is cardiac inefficiency secondary to reduced ATP levels [6]. In a healthy adult, the heart cycles about $6 \mathrm{~kg}$ of ATP every day while beating about 100, 000 times [7]. When ATP synthesis is reduced, such as in DCM disease, the functions of $\mathrm{Ca}^{2+}$ ATPase are impaired, which further disturbs $\mathrm{Ca}^{2+}$ cycling and leads to fatal arrhythmias $[8,9]$. In fact, energy supplements in the form of ATP injections were first used for the treatment of coronary heart disease in the 1940s [10]. In a pathological state, the heart shows a progressive decline in cardiac function along with changes in energy synthesis. Thus, it is important to gain further understanding of energy metabolism from diabetic rat hearts and explore the pathogenesis of DCM.

${ }^{1} \mathrm{H}$ nuclear magnetic resonance (NMR)-based metabonomics has many advantages as a holistic measurement method because it allows dynamic and global changes of specific metabolites in body fluids or tissue samples to be determined-especially those metabolites involved in energy metabolism changes $[11,12]$. Thus, this approach has been used to explore the underlying pathogenesis of diabetes and other metabolic diseases $[13,14]$. We previously used this approach and subsequently observed significant changes in energy metabolites, including higher levels of lipids, ketone bodies, and lactate, in the serum of T2DM rats $[15,16]$. We also have shown that a reduction in energy and the disequilibrium of lipids and methylamine metabolism are closely related to the pathogenic progression of diabetic nephropathy (DN) [17-19]. In the present study, we used quantitative and correlative analysis of ${ }^{1} \mathrm{H}$ NMR spectra of isolated cardiac tissues obtained from diabetic rats to identify the characteristic changes in energy metabolism. This information will allow us to probe into the potential mechanisms of DCM, and provide clues for the development of future therapies.

\section{Materials and Methods}

\section{Subjects}

Male Sprague-Dawley rats with body weights of 160-180 g were purchased from the Shanghai SLAC Laboratory Animal Co. Ltd. and kept in a specific pathogen-free colony of the Laboratory Animal Center of Wenzhou Medical University with regulated temperature and humidity and a 12/12 h light-dark cycle with lights on at 08:00. During the entire experiment, rats were fed standard rat chow and tap water. All animals received care in accordance with the "Guide for the Care and Use of Laboratory Animals" [20]. Procedures using rats were approved by the Institutional Animal Care and Use Committee of Wenzhou Medical University.

\section{Experimental design and sample collection}

Rats were randomly divided into the following six groups: diabetic, 5 weeks; diabetic, 9 weeks; diabetic, 15 weeks; and three corresponding age-matched control groups. After $12 \mathrm{~h}$ of fasting, the rats in the diabetic groups were given an intraperitoneal injection of streptozotocin (STZ, Sigma-Aldrich Corp., St Louis, MO) freshly prepared in citrate buffer $(0.1 \mathrm{M}, \mathrm{pH} 4.5)$ at a single dose of $60 \mathrm{mg} / \mathrm{kg}$ body weight to prepare the diabetic model ( $n=63$, for diabetic screening). Rats in the control groups were injected with the same volume of sodium citrate solvent $(n=45)$. Two days after the injections, the blood glucose concentrations were measured using a tail nick and glucometer (One Touch Ultra, LifeScan Inc., Milpitas, CA), and rats with blood glucose levels higher than $16.70 \mathrm{mmol} / \mathrm{L}$ were classified as diabetic [21].

Cardiac function in anesthetized (4\% chloral hydrate solution $(1 \mathrm{ml} / 100 \mathrm{~g})$ by intraperitoneal injection) diabetic rats and age-matched control rats was evaluated by echocardiography via a Vevo 770 (VisualSonics Inc., Toronto, Ontario, Canada) equipped with a 17-MHz linear array transducer 15 weeks 


\section{Cellular Physiology Cell Physiol Biochem 2018;48:934-946

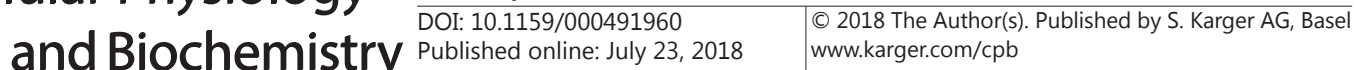 \\ Zhao et al.: Metabolic Alterations in Diabetic Hearts}

after STZ injection. The following indices were obtained: diastolic interventricularseptal thickness (IVSd), diastolic posterior wall thickness (PWd), systolic interventricularseptal thickness (IVSs), systolic posterior wall thickness (PWs), diastolic left ventricular diameter (LVd), systolic left ventricular diameter (LVs), fractional shortening (FS), ejection fraction (EF), and heart rate (HR). Echocardiography was conducted by investigators who were blinded to the experimental groups.

Exactly 5, 9, and 15 weeks after STZ or solvent treatment and after collecting serum samples for NMR analysis, the rats were sacrificed by decapitation. Whole hearts were removed immediately, washed in cold PBS, weighed, snap-frozen in liquid nitrogen, and stored at $-80^{\circ} \mathrm{C}$ until NMR analysis. Some heart tissues from each group were rapidly dissected, fixed overnight in $10 \%$ buffered formalin, embedded in paraffin, sliced into $4 \mathrm{~mm}$ sections, and stained with hematoxylin-eosin (HE) and Masson's trichrome. A blinded pathologist who evaluated all samples for intercellular edema, inflammatory cell infiltration, cardiac fiber degeneration, intracytoplasmic vacuolization, hemorrhage, and mast cell degranulation.

\section{Cardiac metabolite extraction}

The frozen heart tissues were weighed and put into centrifuge tubes. Ice-cold methanol ( $4 \mathrm{~mL} / \mathrm{g})$ and distilled water $(0.85 \mathrm{~mL} / \mathrm{g})$ were added to the tubes. Tissues were homogenized at $4{ }^{\circ} \mathrm{C}$ after thawing, and mixed by vortex. Chloroform $(2 \mathrm{~mL} / \mathrm{g})$ and distilled water $(2 \mathrm{~mL} / \mathrm{g})$ were added to the tubes and mixed again. The tubes were placed on ice for $15 \mathrm{~min}$, and the homogenates centrifuged at $1,000 \mathrm{~g}$ for $15 \mathrm{~min}$ at $4{ }^{\circ} \mathrm{C}$. The supernatants were extracted and lyophilized for about $24 \mathrm{~h}$. The aqueous extracts obtained were then dissolved in $600 \mu \mathrm{L}$ of $99.5 \% \mathrm{D}_{2} \mathrm{O}$ for ${ }^{1} \mathrm{H}$ NMR spectroscopy.

\section{Acquisition of ${ }^{1} H$-NMR spectra and multivariate pattern recognition}

The detailed acquisition protocol of ${ }^{1} \mathrm{H}-\mathrm{NMR}$ spectra is described in our previous reports $[19,22]$. All NMR spectra were phase- and baseline-corrected, and then data-reduced to 1100 integrated regions with widths of $0.01 \mathrm{ppm}$ corresponding to the region of $\delta 10$ to -1 using the Topspin 2.1 software package (Bruker Corp., Karlsruhe, Germany) for multivariate pattern recognition analysis. The region around $\delta 4.69-5.04$ was removed to eliminate artifacts related to the residual water resonance. The remaining spectral segments were normalized to the total sum of the spectral intensity to compensate for variations in the total sample volume. The normalized integral values were then subjected to multivariate pattern recognition analysis using the SIMCA-P ${ }^{+}$V12.0 software package (Umetrics, Umea, Sweden). Following a preliminary principal components analysis, supervised partial least squares-discriminant analysis (PLS-DA) was performed for class discrimination and biomarker identification [23].

Data were visualized with a principal component (PC) scores plot of the first two principal components (PC1 and PC2) to provide the most efficient 2-D representation of the information, where each point represents an individual spectrum of a sample. PLS-DA revealed differences between the groups, which were necessary to eliminate outliers and enhance the quality of the model. The loading plots, which were assessed by the absolute value of the correlation coefficient, $|\mathrm{r}|$, can identify metabolites that contribute to the separation of metabolic profiles. The scores and loading plots complemented each other. A 100 random permutation test was also performed to evaluate the robustness of the PLS-DA model. Meanwhile, two parameters were calculated: $R^{2} X$ and $R^{2} Y$, which explained variance in the matrix of NMR data and class membership, respectively, and $\mathrm{Q}^{2}$, the predictive capability of the model; these are commonly used to indicate the quality of a model [24]. Values of $\mathrm{R}^{2} \mathrm{Y}$ and $\mathrm{Q}^{2}$ close to 1.0 represent an excellent model. Furthermore, the significance of the models was tested by CV-ANOVA in the SIMCA software [25].

\section{Determination of ATP, ADP, and AMP in cardiac tissue extracts}

The cardiac extracts were prepared differently from those described above. Whole rat hearts were immediately snap-frozen in liquid nitrogen without PBS treatment for HPLC analysis. The whole time course was no more than $5 \mathrm{~s}$. Adenine nucleotides (ATP, ADP, and AMP) were analyzed in the aliquots using HPLCUV as per our previous study with minor modifications [17]. Chromatographic separation was achieved on a $250 \mathrm{~mm} \times 4.6 \mathrm{~mm}$ i.d. ZORBAX SB-Aq column bonded to $5-\mu \mathrm{m}$ spherical silica packing (Agilent Inc., Santa Clara, CA). The mobile phase was $99 \% 100 \mathrm{mM} \mathrm{K}_{2} \mathrm{HPO}_{4}-\mathrm{KH}_{2} \mathrm{PO}_{4}$ buffer solution at $\mathrm{pH} 6.0$ and $1 \%$ methanol. The HPLC-UV system was performed at room temperature with a flow rate of $1.0 \mathrm{~mL} \mathrm{~min}^{-1}$. The wavelength of $259 \mathrm{~nm}$ was used for detection and quantification. The identification of different adenine nucleotides was based on the retention times of known amounts of standards. The adenine nucleotides were quantified 


\section{Cellular Physiology Cell Physiol Biochem 2018;48:934-946 \begin{tabular}{c|c} 
DOI: 10.1159/000491960 & $\begin{array}{l}\text { O 2018 The Author(s). Published by S. Karger AG, Basel } \\
\text { www.karger.com/cpb }\end{array}$
\end{tabular} \\ Zhao et al.: Metabolic Alterations in Diabetic Hearts}

using the standard curve of standards in the chromatogram. The results were recorded as millimoles adenine nucleotides per kilogram tissue.

\section{Statistical analysis}

Systemic and statistical metabolic correlation analysis was further applied to display the relationships between certain metabolite integrals, as described previously [26]. Metabolite intensities relative to the sum of the total spectral integral were used as variables, and the magnitude of Pearson's correlation coefficient $(|\mathrm{r}|)$ was calculated in the above-mentioned Java environment. An absolute value of $r$ larger than $0.6(P<0.05)$ is considered to represent a statistically significant relationship between two metabolites. Positive values masked in the pixel map are shown in red and negative values in blue. To determine significant differences between the metabolic levels, SPSS software (Version 13.0, SPSS Inc., Chicago, IL) was used for statistical analysis of the normalized integral values. Comparisons between groups were performed via student's two-tailed t-test or one-way ANOVA followed by Bonferroni correction in the case of multiple comparisons. A $P$-value of less than 0.05 was considered statistically significant. All data shown are means \pm SEM unless otherwise specified.

\section{Results}

Histopathology and cardiac function

Basal HR, LV dimensions, and indices of systolic and diastolic function in both the control and diabetic rats are listed in Table 1 . IVSd, IVSs, PWd, LVd, and LVs were much lower in diabetic rats $(P<0.05$ versus controls), despite the fact that there were no significant differences in PWs between the control and diabetic rats. In addition, lower HR and EF indicators were observed in the diabetic groups than in controls, indicating that the systolic and diastolic functions of the diabetic rats were significantly impaired.

Fig. 1 shows representative histological images of HE- and Masson-stained sections of cardiac muscles from 5-, 9- and 15-week DM and control rats. Regardless of the time points tested, the heart muscles in the control groups showed minimal variations in fiber size and the absence of mononuclear cell infiltration. In contrast, the diabetic rats contained abnormally large fibers, prominent inflammatory cell infiltration, and a high frequency

Table 1. Indicators of cardiac ultrasound function between diabetic and control rats. All values are expressed as means \pm S.D. $n=6$ per group. Abbreviations: IVSd: diastolic interventricularseptal thickness; IVSs: systolic interventricularseptal thickness; PWd: diastolic posterior wall thickness; PWs: systolic posterior wall thickness; LVd: diastolic left ventricular diameter; LVs: systolic left ventricular diameter; FS: fractional shortening; EF: ejection fraction; HR: heart rate. ${ }^{*} \mathrm{P}<0.05$ compared to controls

\begin{tabular}{lcc}
\hline Indicators & Control, 15 weeks & Diabetic, 15 weeks \\
\hline IVSd (mm) & $1.45 \pm 0.10$ & $1.26 \pm 0.07^{*}$ \\
IVSs (mm) & $1.96 \pm 0.10$ & $1.76 \pm 0.13^{*}$ \\
PWd (mm) & $1.50 \pm 0.09$ & $1.17 \pm 0.14^{*}$ \\
PWs (mm) & $2.03 \pm 0.11$ & $1.76 \pm 0.20$ \\
LVd (mm) & $7.50 \pm 0.57$ & $6.88 \pm 0.43^{*}$ \\
LVs $(\mathrm{mm})$ & $3.92 \pm 0.80$ & $3.30 \pm 0.40^{*}$ \\
EF $(\%)$ & $83.55 \pm 6.10$ & $70.83 \pm 4.49^{*}$ \\
FS $(\%)$ & $55.12 \pm 8.82$ & $45.75 \pm 4.13$ \\
HR $(\mathrm{bpm})$ & $441.17 \pm 39.95$ & $402.00 \pm 22.84^{*}$ \\
\hline
\end{tabular}

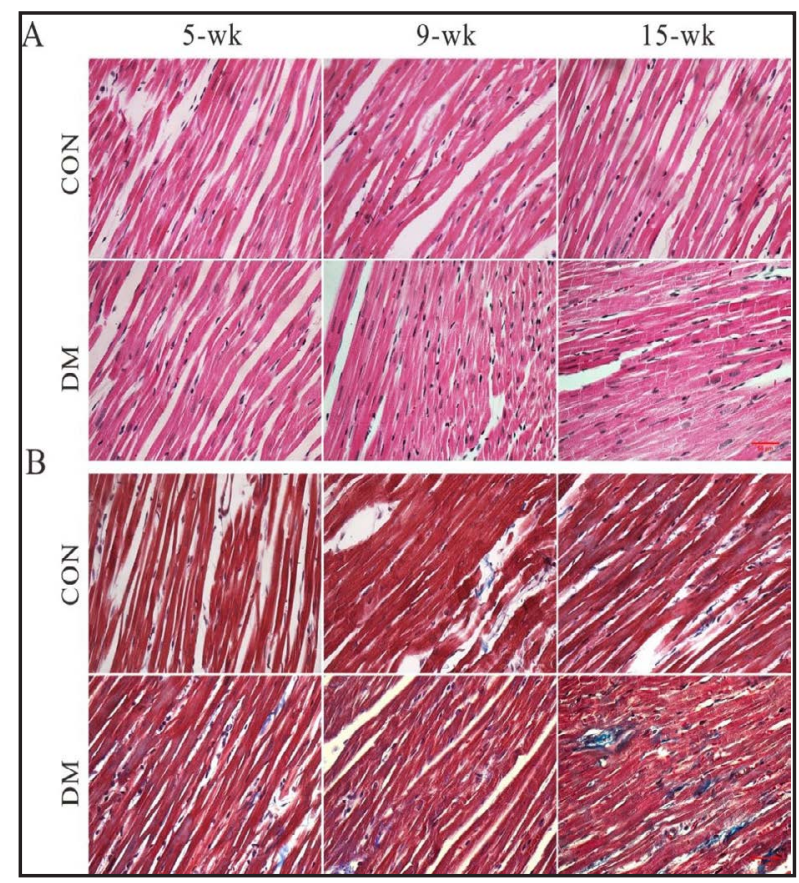

Fig. 1. Representative hematoxylin-eosin (A) and Masson trichrome (B) staining of cardiac tissue from the 5-, 9- and 15 -week diabetic rats and age-matched control rats (200fold, $n=4$ ). 
of centrally nucleated myofibers, especially at the 9- and 15-week time points (Fig. 1A). Fibrosis and muscle calcification were further evaluated with Masson trichrome staining, and we observed that the myocardial fibers had disordered arrangements, as well as large amounts of collagen, in the myocardial interstitium in 15-week diabetic rats (Fig. 1B, Fig. 2). Histopathological data indicated that the diabetic rats progressively developed clear manifestation of cardiac tissue damage during the experimental period.

\section{NMR-based metabonomics}

Representative ${ }^{1} \mathrm{H}$ NMR spectra of cardiac tissue extracts obtained from the diabetic 5-, 9-, and 15-week groups and one group of 15-week control rats are shown in Fig. 3, respectively. From resonance assignments of the metabolites attained in previous studies $[17,27]$ and the $600 \mathrm{MHz}$ library of the Chenomx NMR suite 7.0 (Chenomx Inc., Edmonton, Canada), the metabolites identified included organic acids, sugar, amino acids, organic bases, and purine metabolites, most of which are involved

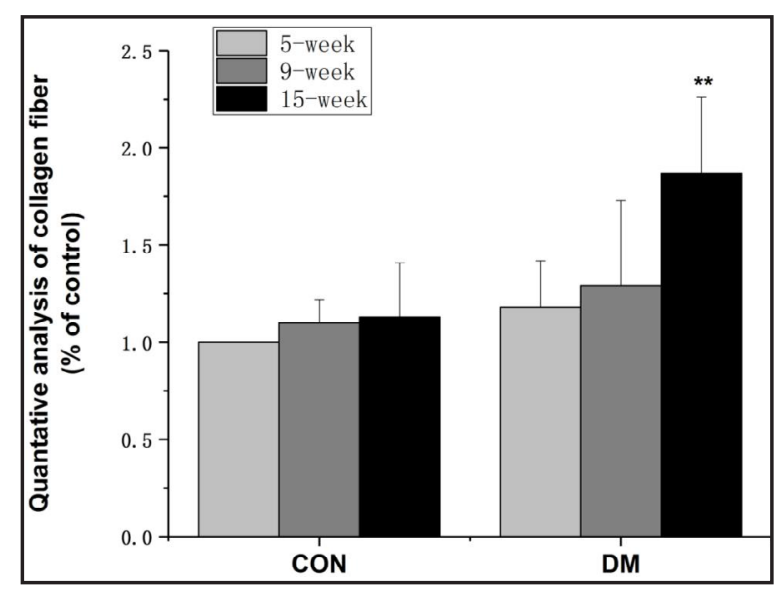

Fig. 2. Comparison of collagen fibrosis in the cardiac tissues from different groups of rats. ${ }^{* *} \mathrm{P}<0.01$ compared with age-matched controls by independent samples t-test.

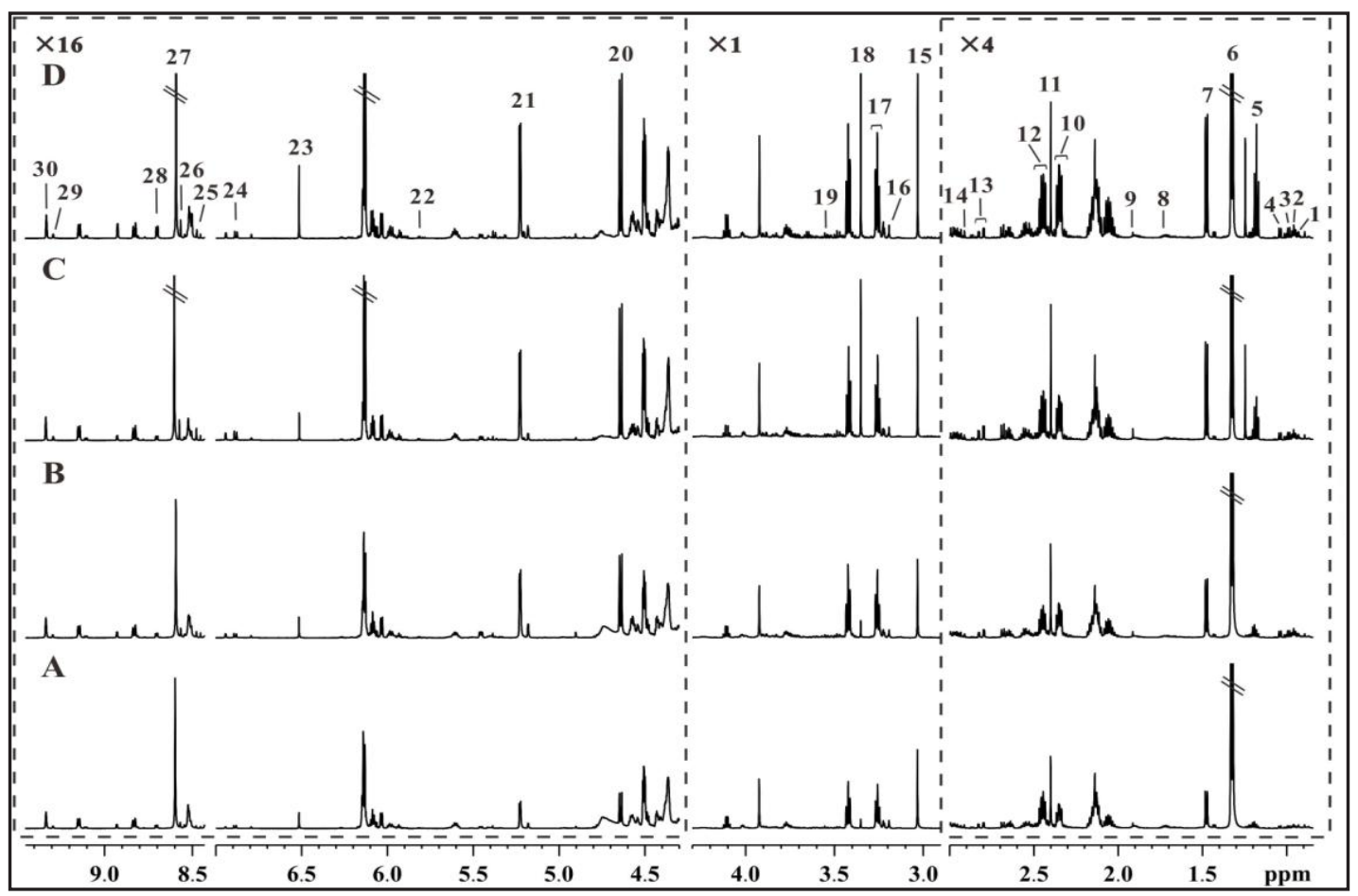

Fig. 3. Typical ${ }^{1} \mathrm{H}$ NMR spectra of cardiac extracts from control rats at 5 weeks (A), and diabetic rats at 5 (B), 9 (C) and 15 (D) weeks. Key: 1, pantothenate; 2, leucine; 3, isoleucine; 4, valine; 5, 3-hydroxybutyrate; 6, lactate; 7, alanine; 8 , arginine; 9 , acetate; 10 , glutamate; 11 , succinate; 12 , glutamine; 13, 2-ketoglutarate; 14 , aspartate; 15 , trimethylamine; 16 , creatine; 17 , choline; 18 , taurine; 19 , methanol; 20 , glycine; $21, \beta$-glucose; 22, $\alpha$-glucose; 23 , uracil; 24 , fumarate; 25 , tyrosine; 26 , formate; $27, \mathrm{ATP}+\mathrm{ADP}+\mathrm{AMP}$ (AXP); 28 , niacinamide; $29, \mathrm{NAD}^{+} ; 30, \mathrm{NADP}^{+}$.

\section{KARGER}


in energy or glucose metabolism pathways.

The PLS-DA score plots of the spectra showed distinct, easily detectable differences between the diabetic rats and control rats, at weeks 5, 9 and 15 after STZ treatment, suggesting substantial differences in metabolic profiles during the progression of diabetes (Fig. 4). The parameters for evaluation of the PLS-DA model validity, R2Y, Q2, and the P values for each model showed that the PLS-DA models were robust and credible (Table 2). The loading plots corresponding to the score plots showed that during the progression of diabetes, glucose, valine, leucine, isoleucine, 3-hydroxybutyrate (3-HB), glutamate, and taurine levels were elevated, and those of ATP/ADP/AMP (AXP), lactate, 2 -ketoglutarate $(\alpha-K G)$, creatine, choline, and glutamine were reduced.

Table 3 summarizes the $|r|$ values of metabolites accounting for PLS-DA separation models of cardiac extracts and lists the results from the statistical analysis for comparison. The trends in metabolite changes indicated by the PLS-DA loading plots were in good agreement with those obtained by statistical analysis. The lower levels of several energy metabolites (highenergy phosphate pool, i.e., AXP, and creatine) were in accordance with the progression of diabetes (Fig. 5). Representing the main pathways of glucose metabolism, the levels of glycolysis-related product (lactate) and TCA cycle intermediates (succinate and 2-oxoglutarate) were obviously reduced at most of the time points studied. Levels of branchedchained amino acids (BCAAs, including valine, leucine, and isoleucine), alanine, and glycine

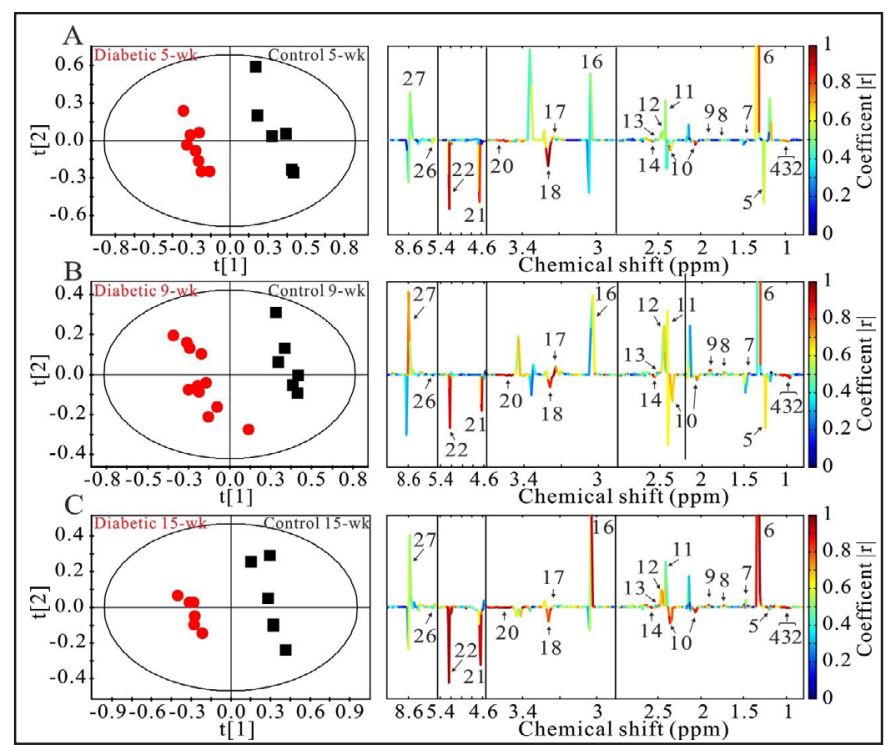

Fig. 4. Multivariate pattern recognition analysis of cardiac extracts from diabetic and control rats at different time points. Projection of latent structure-discriminant analysis score plots (left) and corresponding coefficient-coded loading plots (right) based on cardiac ${ }^{1} \mathrm{H}$ NMR spectra at 5 weeks (A), 9 weeks (B), and 15 weeks (C) after STZ or vehicle treatment, respectively. The key for the metabolites is the same as in Fig. 3.

Table 2. Cross-validated data of PLS-DA models in diabetic rats compared to age-matched controls

\begin{tabular}{lccccc}
\hline Time & $\mathrm{N}$ & $\mathrm{R}^{2} \mathrm{X}$ & $\mathrm{R}^{2} \mathrm{Y}$ & $\mathrm{Q}^{2}$ & $\mathrm{P}$ \\
\hline $5 \mathrm{w}$ & 14 & 0.55 & 0.98 & 0.93 & 0.00 \\
$9 \mathrm{w}$ & 18 & 0.55 & 0.96 & 0.91 & 0.00 \\
$15 \mathrm{w}$ & 12 & 0.66 & 0.98 & 0.94 & 0.00 \\
\hline
\end{tabular}

Table 3. Values of $|\mathrm{r}|$ for metabolites accounting for PLS-DA models of cardiac extracts and statistical analysis to compare diabetes and control rats. Abbreviations: AXP, high-energy phosphate pool (ATP+ADP+AMP)

\begin{tabular}{|c|c|c|c|c|c|c|c|}
\hline \multirow[t]{2}{*}{ No. } & \multirow[t]{2}{*}{ Metabolites } & \multicolumn{2}{|c|}{$5 w$ Diabetes versus control } & \multicolumn{2}{|c|}{$\begin{array}{l}9 \mathrm{w} \text { Diabetes } \\
\text { versus control }\end{array}$} & \multicolumn{2}{|c|}{$\begin{array}{l}15 \mathrm{w} \text { Diabetes } \\
\text { versus control }\end{array}$} \\
\hline & & $r$ value & Fold change & rvalue & Fold change & $\mathrm{r}$ value & Fold change \\
\hline 1 & Pantothenate & 0.13 & -0.21 & 0.21 & -0.04 & 0.67 & -0.09 \\
\hline 2 & Leucine & 0.70 & $0.63^{*}$ & 0.74 & $0.84^{*}$ & 0.80 & $0.75^{*}$ \\
\hline 3 & Isoleucine & 0.73 & $0.52^{*}$ & 0.83 & $0.77^{*}$ & 0.85 & $0.74^{*}$ \\
\hline 4 & Valine & 0.59 & $0.72^{*}$ & 0.82 & $0.91^{*}$ & 0.87 & $0.97^{*}$ \\
\hline 5 & 3-hydrobutyrate & 0.37 & 0.16 & 0.46 & $0.38^{*}$ & 0.64 & $0.91^{*}$ \\
\hline 6 & $\begin{array}{l}\text { Lactate } \\
\text {. }\end{array}$ & 0.91 & $-0.30^{*}$ & 0.88 & $-0.29^{*}$ & 0.96 & $-0.32^{*}$ \\
\hline 7 & Alanine & 0.14 & 0.02 & 0.44 & 0.12 & 0.39 & -0.04 \\
\hline 8 & Arginine & 0.47 & -0.09 & 0.69 & $-0.08^{*}$ & 0.51 & -0.13 \\
\hline 9 & Acetate & 0.81 & $-0.12^{*}$ & 0.85 & $-0.10^{*}$ & 0.76 & $-0.13^{*}$ \\
\hline 10 & Glutamate & 0.79 & $0.21^{*}$ & 0.69 & $0.21^{*}$ & 0.84 & $0.37^{*}$ \\
\hline 11 & Succinate & 0.35 & -0.14 & 0.69 & $0.05^{*}$ & 0.26 & -0.18 \\
\hline 12 & Glutamine & 0.64 & $-0.18^{*}$ & 0.50 & $-0.18^{*}$ & 0.64 & $-0.19^{*}$ \\
\hline 13 & 2-Oxoglutarate & 0.17 & -0.17 & 0.48 & -0.18 & 0.81 & $-0.19^{*}$ \\
\hline 14 & Aspartate & 0.33 & -0.20 & 0.22 & 0.01 & 0.66 & $-0.07^{*}$ \\
\hline 15 & Trimethylamine & 0.09 & 0.07 & 0.71 & $0.20^{*}$ & 0.18 & -0.05 \\
\hline 16 & Creatine & 0.39 & -0.07 & 0.49 & -0.20 & 0.86 & $-0.28^{*}$ \\
\hline 17 & Choline & 0.54 & -0.13 & 0.48 & -0.08 & 0.66 & $-0.11^{*}$ \\
\hline 18 & Taurine & 0.96 & $0.33^{*}$ & 0.83 & $0.15^{*}$ & 0.81 & $0.14^{*}$ \\
\hline 20 & Glycine & 0.49 & 0.11 & 0.76 & $0.13^{*}$ & 0.77 & $0.31^{*}$ \\
\hline 21 & $\beta$-glucose & 0.68 & $0.80^{*}$ & 0.80 & $0.68^{*}$ & 0.85 & $0.91^{*}$ \\
\hline 23 & Uracil & 0.12 & -0.18 & 0.35 & 0.01 & 0.47 & 0.01 \\
\hline 24 & Fumarate & 0.10 & 0.07 & 0.04 & -0.25 & 0.07 & -0.07 \\
\hline 25 & Tyrosine & 0.16 & -0.16 & 0.16 & 0.07 & 0.84 & -0.09 \\
\hline 26 & Formate & 0.31 & 0.08 & 0.60 & $0.40^{*}$ & 0.62 & 0.47 \\
\hline 27 & AXP & 0.67 & $-0.08^{*}$ & 0.77 & $-0.13^{*}$ & 0.78 & $-0.15^{*}$ \\
\hline 28 & Niacinamide & 0.47 & -0.01 & 0.09 & -0.32 & 0.50 & 0.05 \\
\hline 29 & $\mathrm{NAD}^{+}$ & 0.28 & 0.05 & 0.16 & 0.09 & 0.65 & $-0.14^{*}$ \\
\hline 30 & $\mathrm{NADP}^{+}$ & 0.46 & 0.11 & 0.87 & $0.16^{*}$ & 0.79 & $0.02^{*}$ \\
\hline
\end{tabular}


were clearly higher in diabetic rats, while glutamine levels were lower, which suggested that altered amino acid metabolism is associated with DCM. In addition, significant levels of $\mathrm{NAD}^{+}$and $\mathrm{NADP}^{+}$, which are closely related to oxide-reduction reaction in the body, showed metabolic changes, especially in the 15week diabetic group.

To define the specific metabolic changes at the local level in the heart, serum samples from the 5-, 9- and 15week diabetic and control rats were also analyzed (Table 4). We identified many metabolic changes in serum samples but these were not consistent with the results of isolated cardiac extracts (Table 5). For example, the decreases in leucine, isoleucine, valine, 3-HB, glycine in serum samples of diabetic 5-weekold rats were in contrast to the cardiac results. Moreover, we found no changes in tyrosine, alanine, acetate, aspartate, or formate levels in the serum samples, which again differed to the cardiac data. However, similar trends in changes in several metabolites such as glutamine, creatine, and choline were revealed in both serum and heart tissues. Nevertheless, the results provided partial confirmation of the characteristic changes in DCM.

To determine the individual levels of AMP, ADP, and ATP in the adenine nucleotide pool, AXP, the cardiac tissues were further investigated by HPLC technology (Fig. 6). Compared with the age-matched control rats, significant decreases in ATP and ADP concentrations $(\mathrm{P}<0.05)$ were observed,

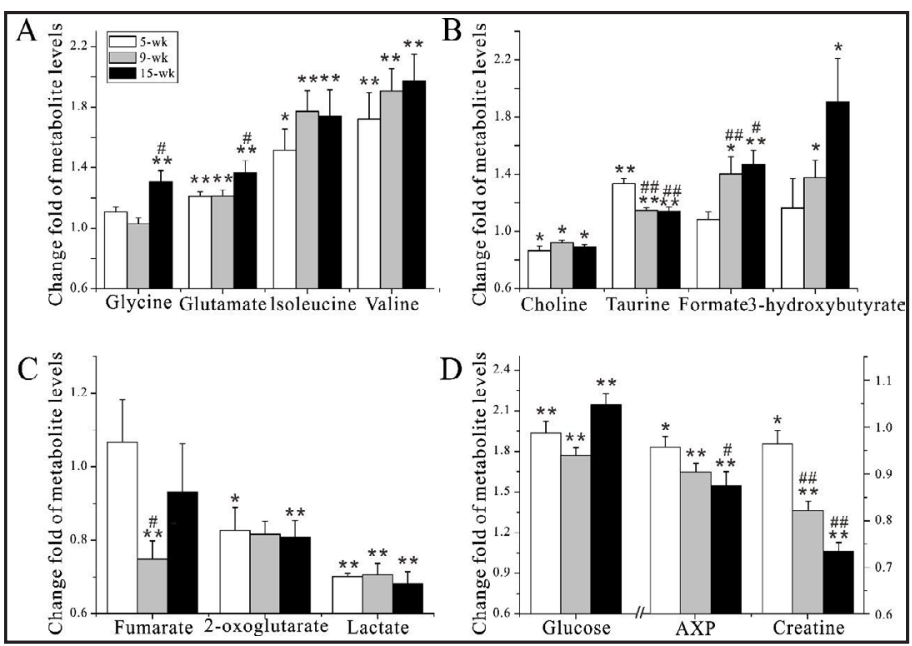

Fig. 5. Fold changes of metabolite levels in cardiac ${ }^{1} \mathrm{H}$ NMR spectra from diabetic groups compared to age-matched control groups. The results are expressed as means $\pm \mathrm{SE}$. ${ }^{*} \mathrm{P}<0.05$ and ${ }^{* *} \mathrm{P}<0.01$ compared with age-matched control groups by an independent samples t-test; ${ }^{\#} \mathrm{P}<0.05$ and ${ }^{\# \#} \mathrm{P}<0.01$ compared with the 5 week diabetic group.

Table 4. Values of $|\mathrm{r}|$ for metabolites accounting for PLS-DA models of serum samples and statistical analysis for comparison between diabetic and age-matched control rats. ${ }^{*} \mathrm{P}<0.05$ compared to controls

\begin{tabular}{|c|c|c|c|c|c|c|c|}
\hline \multirow{2}{*}{ No } & \multirow{2}{*}{ Metabolites } & \multirow{2}{*}{$\begin{array}{c}5 W \text { Diabetes } \\
\text { versus control } \\
r \text { value }\end{array}$} & \multirow[b]{2}{*}{$\begin{array}{c}\text { Fold } \\
\text { change }\end{array}$} & \multicolumn{2}{|c|}{$\begin{array}{l}9 \mathrm{~W} \text { Diabetes } \\
\text { versus control }\end{array}$} & \multicolumn{2}{|c|}{$\begin{array}{l}15 W \text { Diabetes } \\
\text { versus control }\end{array}$} \\
\hline & & & & r value & $\begin{array}{l}\text { Fold } \\
\text { change }\end{array}$ & $r$ value & $\begin{array}{c}\text { Fold } \\
\text { change }\end{array}$ \\
\hline 1 & Formate & 0.38 & 0.15 & 0.30 & 0.09 & 0.13 & 0.21 \\
\hline 2 & Phenylabnine & 0.06 & 0.08 & 0.70 & $-0.27^{\circ}$ & 0.03 & 0.13 \\
\hline 3 & Tyrosine & 0.34 & -0.13 & 0.39 & -0.12 & 0.18 & -0.06 \\
\hline 4 & Histidine & 0.01 & 0.03 & 0.73 & $-0.40^{\circ}$ & 0.21 & 0.24 \\
\hline 5 & Allantoin & 0.66 & $0.17^{\circ}$ & 0.18 & 0.04 & 0.60 & $0.58^{*}$ \\
\hline 6 & Creatine & 0.51 & 0.13 & 0.67 & $-0.33^{\circ}$ & 0.48 & $-0.05^{\circ}$ \\
\hline 7 & Glycine & 0.28 & -0.02 & 0.80 & $-0.27^{\circ}$ & 0.14 & -0.04 \\
\hline 8 & Aspartate & 0.46 & 0.13 & 0.61 & $-0.06^{\circ}$ & 0.55 & $-0.10^{\circ}$ \\
\hline 9 & Dimethylamine & 0.38 & 0.07 & 0.65 & $0.21^{\circ}$ & 0.07 & 0.03 \\
\hline 10 & Citrate & 0.45 & 0.11 & 0.51 & $0.13^{\circ}$ & 0.40 & 0.24 \\
\hline 11 & Gilutamine & 0.61 & $-0.21^{\circ}$ & 0.41 & $-0.31^{\circ}$ & 0.44 & $-0.21^{\circ}$ \\
\hline 12 & Pyruvate & 0.56 & $-0.33^{\circ}$ & 0.58 & $-0.28^{\circ}$ & 0.68 & $0.09^{\circ}$ \\
\hline 13 & $\begin{array}{c}3- \\
\text { hydrobutyrate }\end{array}$ & 0.27 & -0.50 & 0.36 & -0.23 & 0.54 & -0.17 \\
\hline 14 & Acetoacetate & 0.07 & 0.11 & 0.13 & -0.02 & 0.65 & $0.41^{\circ}$ \\
\hline 15 & Acetone & 0.58 & $-0.24^{\circ}$ & 0.35 & -0.02 & 0.14 & -0.28 \\
\hline 16 & Acetate & 0.75 & $0.42^{\circ}$ & 0.51 & $0.49^{\circ}$ & 0.65 & $0.24^{\circ}$ \\
\hline 17 & Alanine & 0.27 & 0.02 & 0.74 & $-0.12^{*}$ & 0.69 & -0.24 \\
\hline 18 & Lactate & 0.05 & -0.08 & 0.42 & $-0.21^{\circ}$ & 0.56 & $-0.13^{\circ}$ \\
\hline 19 & Valine & 0.82 & $0.32^{\circ}$ & 0.47 & $-0.20^{\circ}$ & 0.49 & $-0.55^{\circ}$ \\
\hline 20 & Isoleucine & 0.74 & $0.23^{\circ}$ & 0.25 & $-0.26^{\circ}$ & 0.49 & $-0.41^{\circ}$ \\
\hline 21 & Leucine & 0.65 & $0.23^{\circ}$ & 0.50 & $-0.13^{\circ}$ & 0.60 & -0.46 \\
\hline 22 & LDL/VLDL & 0.66 & $1.03^{\circ}$ & 0.62 & $0.10^{\circ}$ & 0.61 & $0.18^{\circ}$ \\
\hline
\end{tabular}

Table 5. Fold changes in metabolite levels of serum samples and heart from diabetic rats compared to age-matched control rats. ${ }^{*} \mathrm{P}<0.05$ compared to controls

\begin{tabular}{|c|c|c|c|c|c|c|c|}
\hline \multirow[t]{2}{*}{ No } & \multirow[t]{2}{*}{ Metabolites } & \multicolumn{2}{|c|}{$\begin{array}{c}5 \mathrm{~W} \text { Diabetes } \\
\text { versus control }\end{array}$} & \multicolumn{2}{|c|}{$\begin{array}{c}9 \mathrm{~W} \text { Diabetes } \\
\text { versus control }\end{array}$} & \multicolumn{2}{|c|}{$\begin{array}{l}\text { 15W Diabetes } \\
\text { versus control }\end{array}$} \\
\hline & & Serum & Heart & Serum & Heart & Serum & Heart \\
\hline 1 & Formate & 0.15 & 0.08 & 0.09 & 0.40 & 0.21 & $0.47^{*}$ \\
\hline 2 & Tyrosine & -0.13 & -0.16 & -0.12 & 0.07 & -0.06 & $-0.09^{*}$ \\
\hline 3 & Creatine & 0.13 & -0.07 & $-0.33^{*}$ & $-0.20^{*}$ & $-0.05^{*}$ & $-0.28^{*}$ \\
\hline 4 & Glycine & -0.02 & $0.11^{*}$ & $-0.27^{*}$ & $0.13^{*}$ & -0.04 & $0.31^{*}$ \\
\hline 5 & Aspartate & 0.13 & -0.20 & $-0.06^{*}$ & 0.01 & $-0.10^{*}$ & $-0.07^{*}$ \\
\hline 6 & Glutamine & $-0.21^{*}$ & $-0.18^{*}$ & $-0.31^{*}$ & $-0.18^{*}$ & $-0.21^{*}$ & $-0.19^{*}$ \\
\hline 7 & 3-hydrobutyrate & -0.50 & 0.16 & $-0.23^{*}$ & $0.38^{*}$ & $-0.17^{*}$ & $0.91^{*}$ \\
\hline 8 & Acetate & $0.42^{*}$ & $-0.12^{*}$ & $0.49^{*}$ & $-0.10^{*}$ & $0.24^{*}$ & $-0.13^{*}$ \\
\hline 9 & Alanine & -0.05 & 0.02 & $-0.12^{*}$ & 0.12 & $-0.24^{*}$ & -0.04 \\
\hline 10 & Lactate & -0.08 & $-0.30^{*}$ & $-0.21^{*}$ & $-0.29^{*}$ & $-0.13^{*}$ & $-0.32^{*}$ \\
\hline 11 & Valine & $0.32^{*}$ & $0.72^{*}$ & $-0.20^{*}$ & $0.91^{*}$ & $-0.55^{*}$ & $0.97^{*}$ \\
\hline 12 & Isoleucine & $0.23^{*}$ & $0.52^{*}$ & $-0.26^{*}$ & $0.77^{*}$ & $-0.41^{*}$ & $0.74^{*}$ \\
\hline 13 & Leucine & $0.23^{*}$ & $0.63^{*}$ & $-0.13^{*}$ & $0.84^{*}$ & $-0.46^{*}$ & $0.75^{*}$ \\
\hline
\end{tabular}


especially in diabetic rats at weeks 9 and 15, while there were no changes in AMP concentrations or ATP/ ADP ratios (Fig. 7). Thus, the trend relating to changes in individual levels of the adenine nucleotide pool reflected the progressively lower AXP levels determined by NMR.

\section{Correlation analysis}

AXP, creatine, and glucose are well-known markers of energy metabolites in organisms. In particular, AXP and creatine constitute the high-energy phosphate pool.In diabetic rats, AXP and creatine levels in cardiac extracts progressively decreased while glucose increased. Using Pearson's correlation analysis of AXP and glucose in 15 week diabetic rats, we ascertained that the pathways associated with energy and glucose metabolism were disrupted (Fig. 8, Table 6).

Remarkably, in the cardiac extracts of control rats, AXP showed positive correlations with lactate, 2-KG, succinate, alanine, and glutamine, accompanied by a negative correlation with 3-HB and glucose. No correlations

Fig. 7. Comparison of adenine nucleotide concentrations in cardiac extracts from diabetic rats at different times. ${ }^{*} \mathrm{P}<0.05$ compared with age-matched controls by independent samples t-test.

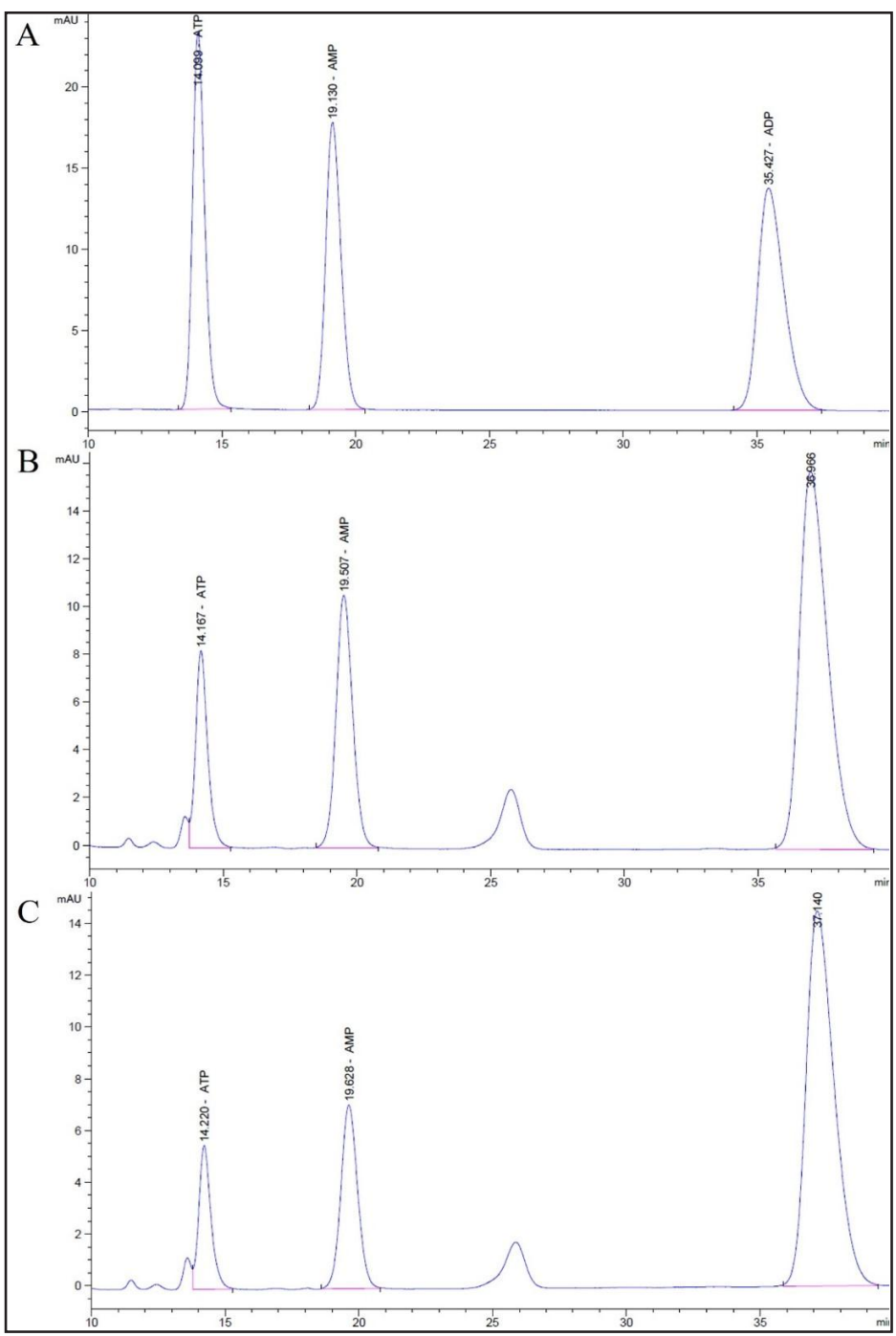

Fig. 6. HPLC chromatograms of standard adenine nucleotides (A) in cardiac extracts from control at 15 weeks (B) and diabetic rats at 15 weeks (C). In A, the retention times were as follows: ATP, $14.0 \mathrm{~min}$; ADP, $19.1 \mathrm{~min}$; and AMP, $35.4 \mathrm{~min}$.

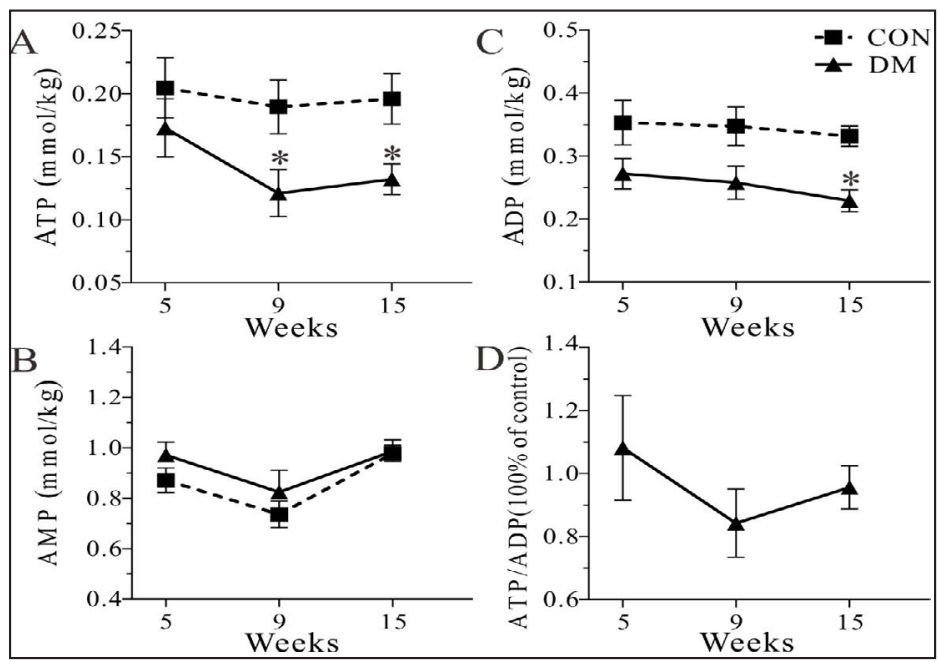


between AXP and leucine, isoleucine, or valine were found. The glucose levels in the hearts of control rats displayed a negative relationship with lactate, succinate, 2-KG, valine, isoleucine, alanine, and glutamine and were accompanied by a positive correlation with 3-HB and glycine. However, most of these associations displayed in the controls were not found in the heart tissues of diabetic rats.

\section{Disrupted metabolic pathways}

Substances found in the pathways involved in energy metabolism, including the TCA cycle, glycolysis, fatty acid $\beta$-oxidation, and different amino acids, during the progression of DCM were drawn according to the KEGG database (http://www.genome.jp/kegg/ pathway.html). For example, reduced concentrations of succinate, fumarate, and $\alpha-K G$ found in the diabetic hearts suggested down-regulation of the TCA cycle (Fig. 9). Moreover, the elevated levels of 3-HB indicated fast fatty acid $\beta$-oxidation and ketogenesis in the diabetic hearts. Thus, a combination that included assessment of the metabolic changes and correlation analysis provided clearer insight into the detailed energy metabolism pathways involved in the progression of DCM.

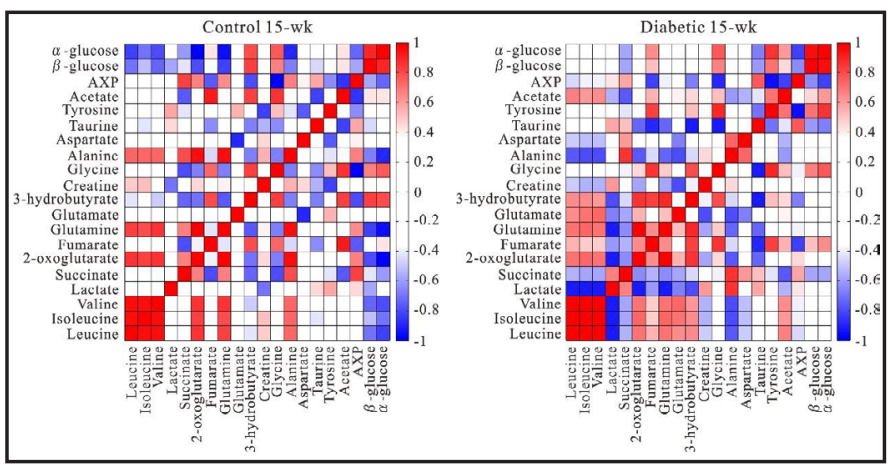

Fig. 8. Pearson's correlations of the quantities of metabolites determined in cardiac extracts from 15 week control and diabetic rats. Red and blue represent positive and negative correlations, respectively, while the color scale represents Pearson's correlation coefficients.

Table 6. Correlation analysis of glucose and AXP levels. ${ }^{\text {aChanges }}$ in diabetic heart extracts compared to age-matched controls. ${ }^{b}$ Correlation analysis of 15 week in control rats $\rightarrow$ with 15 -week in diabetic rats. Abbreviations: BCAAs: branched-chain amino acids (leucine, isoleucine, valine); 3-HB: 3-hydrobutyrate; 2-KG: 2-oxoglutarate; AXP: high-energy phosphate pool (ATP+ADP+AMP)

\begin{tabular}{lcccc}
\hline Compounds & $\begin{array}{c}\text { Alterations in } \\
\text { levels }\end{array}$ & Correlation with glucose & Correlation with AXPb & Relationship with glucose or AXP metabolism \\
\hline Lactate & Decreased & Negative $\rightarrow$ none & Positive $\rightarrow$ positive & Glycolysis attenuation, ATP reduction \\
Succinate & Decreased & Negative $\rightarrow$ none & Positive $\rightarrow$ positive & TCA cycle attenuation, ATP reduction \\
2-KG & Decreased & Negative $\rightarrow$ none & Positive $\rightarrow$ positive & TCA cycle attenuation, ATP reduction \\
3-HB & Increased & Positive $\rightarrow$ none & Negative $\rightarrow$ negative & Lipid metabolism, ATP production \\
BCAAs & Increased & Negative $\rightarrow$ none & None $\rightarrow$ negative & ATP reduction \\
Alanine & Increased & Negative $\rightarrow$ none & Positive $\rightarrow$ none & ATP reduction \\
Glutamine & Decreased & Negative $\rightarrow$ none & Positive $\rightarrow$ none & ATP reduction \\
Glycine & Increased & Positive $\rightarrow$ positive & Negative $\rightarrow$ none & ATP production \\
Glucose & Increased & Null & Negative $\rightarrow$ negative & Glucose metabolism attenuation, ATP reduct \\
\hline
\end{tabular}

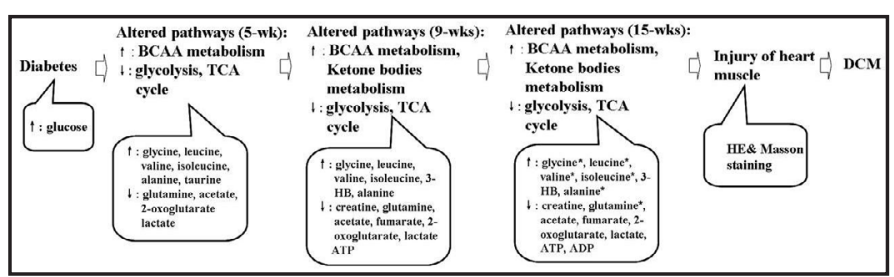

Fig. 9. Schematic diagram of pathogenesis in DCM rats treated with a single dose of streptozotocin. Increased or decreased metabolite levels compared with control rats are indicated by adjoining arrows.

\section{Discussion}

Diabetes is associated with changes in myocardial substrate metabolism, which is believed to contribute to cardiac dysfunction. Increasing evidence indicates that dysfunction of mitochondrial energy metabolism plays a crucial role in the pathogenesis of DCM [9]. Mitochondrial uncoupling may represent one mechanism alongside increased oxidative stress and ROS production, that leads to reduced cardiac efficiency and lower ATP generation in diabetic hearts [28-30]. In addition, defective energy metabolism in the heart is likely to impair energy-requiring processes such as protein synthesis, maintenance of transmembrane 
$\mathrm{Na}^{+}$and $\mathrm{Ca}^{2+}$ gradients, and resting acto-myosin ATPase in the heart [31]. Furthermore, the upregulation of MAPK signal transduction can improve both cardiac energy metabolism and cardiac function [32].

We previously reported a decline in ATP levels in the renal tissues of diabetic nephropathy rats [17]. Similarly, several other studies have shown that ATP and ATP synthase in the mitochondria of diabetic hearts is down-regulated [2,33, 34]. In the present study, continually decreasing levels of the high-energy phosphate pool in the DCM rats were confirmed by NMR and HPLC. HE- and Masson's-stained sections of cardiac muscle, alongside cardiac ultrasound showed that the diabetic rats developed clear cardiac dysfunction, time-dependently. Therefore, impaired energy synthesis was accompanied by the onset and progression of cardiac damage during the evolution of diabetes. Systemic and holistic descriptions of the changes in energy metabolism in DCM rats are of great importance and will enable a comprehensive understanding of the pathogenic mechanisms.

Correlation analysis of the metabolites in cardiac tissues of 15-week-old diabetic rats and controls was performed to gain insight into the changes in energy metabolism associated with DCM. Positive or negative correlation indicates direct or indirect relationships among the metabolites (in one metabolic pathway). In a pathological state, these relationships disappear, which means that the metabolic pathway is attenuated or interrupted $[18,26]$. In our study, we found positive correlations of AXP with lactate, succinate, $\alpha-K G$, and fumarate, and a negative correlation of AXP with glucose in the cardiac tissues from both diabetic rats and controls, which suggested that energy metabolites were in both cases derived from the TCA cycle and glycolysis pathways, regardless of the hyperglycemic or physiological statuses. Conversely, the lack of correlation between AXP and several amino acids indicated that the diabetic hearts barely produced energy from amino acid metabolic pathways in contrast to healthy hearts.

To further examine the changes in glucose metabolism in DCM, we analyzed correlations between the metabolites identified and glucose levels. Glucose homeostasis in control rats was revealed from positive or negative correlations between glucose and products of glucose metabolism, namely, lactate, TCA cycle intermediates, and several amino acids, namely, glutamine, glycine, BCAAs, and alanine. However, most of these correlations were dramatically different or missing in diabetic hearts, which means that most glucose metabolic pathways were attenuated or suppressed in the disease state (except for glycine). In addition, glycine levels were increased in diabetic hearts, which strongly suggested enhanced glycine metabolism in DCM conditions.

Substantial clinical evidence shows that long-term hyperglycemia is associated with worse cardiovascular outcome $[35,36]$. Hyperglycemia leads to the accumulation of advanced glycation end-products. The mechanisms that mediate hyperglycemia-induced tissue and mitochondrial damage are mitochondrial DNA mutation, over-activity of the hexosamine pathway, and the activation of protein kinase C [9]. In this study, glycolysis, the TCA cycle, and the metabolism of some amino acids were down-regulated in rats with DCM, which resulted in reduced glucose utilization and less ATP production. Thus, this finding indicates that the pathways related to energy synthesis may be responsible for cardiac dysfunction.

The enhanced 3-HB observed in this study suggested fast ketogenesis and lipid metabolism in diabetic hearts. Lipids and ketone bodies have been implicated in the onset of insulin resistance in the myocardium, cellular damage, and contractile dysfunction $[37,38]$. It is known that both ketone bodies and glucose are major metabolic substrates and fuels that heart contractions in a normal physiological state [9] but our results suggested that cells switched to ketogenesis to maintain energy homeostasis following the loss of energy caused by impaired glucose oxidases in the diabetic state.

In diabetic hearts, elevated BCAA levels appear to protect the heart against myocardial injury by improving systolic and diastolic myocardial function in a hyperglycemic environment [39], which has also been suggested to affect insulin sensitivity by activating targets of rapamycin complex (mTORC) and downstream ribosomal protein S6 kinase 1 (S6K1) [40-43]. 


\section{Cellular Physiology Cell Physiol Biochem 2018:48:934-946 \begin{tabular}{l|l} 
DOI: 10.1159/000491960 & $\begin{array}{l}\text { O } 2018 \text { The Author(s). Published by S. Karger AG, Basel } \\
\text { www.karger.com/cpb }\end{array}$
\end{tabular} \\ Zhao et al.: Metabolic Alterations in Diabetic Hearts}

On the other hand, BCAAs can provide materials that drive energy production, and they play important roles in the maintenance and growth of muscle $[44,45]$. In the BCAA catabolic pathway, BCAAs are first converted into branched-chain alpha-ketoacids (BCKAs) by branched-chain amino-transferase (BCAT), followed by a decarboxylation reaction via branched-chain alpha-ketoacid dehydrogenase (BCKDH) complex, and eventually metabolized to acetyl-CoA for oxidation in the TCA cycle [46]. Recently, it has been reported that chronic accumulation of BCAAs downregulates the hexosamine biosynthetic pathway and inactivates pyruvate dehydrogenase, which is accompanied by decreases in glucose uptake, and protein glycosylation [46]. The results of that study identified a novel mechanism that enables defective BCAA catabolism to suppress glucose metabolism and sensitize the heart to hyperglycemic injury. Therefore, a critical role of BCAA catabolism is in the regulation of cardiac glucose or energy metabolism was revealed.

In this study, we revealed that the inhibition of glycolysis and the TCA cycle enhanced lipid metabolism and altered the levels of amino acids. These events were closely associated with a decline in energy production in DCM rats and played a crucial role in the pathogenesis of DCM. However, the causal relationship between the changes in energy metabolism and DCM should be investigated using other approaches, such as genomics or signal transduction, to advance our understanding of the mechanisms of DCM and promote early clinical diagnosis and treatment.

\section{Acknowledgements}

This work was supported by the National Natural Science Foundation of China (Nos. 81770830, 21575105, and 81400863), Zhejiang Provincial Natural Science Foundation (Nos. LY17H160049 and LQ18H160027) and Science and Technology Program of Wenzhou Municipal (No. Y20160524).

\section{Disclosure Statement}

The authors declare no competing financial interests.

\section{References}

1 Diao CF, Zhao LC, Guan MM, Zheng YQ, Chen MJ, Yang YJ, Lin L, Chen WJ, Gao HC: Systemic and characteristic metabolites in the serum of streptozotocin-induced diabetic rats at different stages as revealed by a H-1-NMR based metabonomic approach. Mol Biosyst 2014;10:686-693.

-2 Liang Z, Leo S, Wen H, Ouyang M, Jiang W, Yang K: Triptolide improves systolic function and myocardial energy metabolism of diabetic cardiomyopathy in streptozotocin-induced diabetic rats. BMC Cardiovasc Disord 2015;15:42.

3 Devereux RB, Roman MJ, Paranicas M, O'Grady MJ, Lee ET, Welty TK, Fabsitz RR, Robbins D, Rhoades ER, Howard BV: Impact of diabetes on cardiac structure and function - The strong heart study. Circulation 2000;101:2271-2276.

-4 Boudina S, Abel ED: Diabetic cardiomyopathy revisited. Circulation 2007;115:3213-3223.

5 Feuvray D, Darmellah A: Diabetes-related metabolic perturbations in cardiac myocyte. Diabetes Metab 2008;34:S3-9.

6 Taha M, Lopaschuk GD: Alterations in energy metabolism in cardiomyopathies. Ann Med 2007;39:594-607.

7 Neubauer S: The failing heart--an engine out of fuel. N Engl J Med 2007;356:1140-1151.

8 Liu T, Brown DA, O’Rourke B: Role of mitochondrial dysfunction in cardiac glycoside toxicity. J Mol Cell Cardiol 2010;49:728-736.

-9 Aon MA, Tocchetti CG, Bhatt N, Paolocci N, Cortassa S: Protective Mechanisms of Mitochondria and Heart Function in Diabetes. Antioxid Redox Sig 2015;22:1563-1586. 


\section{Cellular Physiology Cell Physiol Biochem 2018;48:934-946 \begin{tabular}{l|l} 
DOI: 10.1159/000491960 & $\begin{array}{l}\text { O } 2018 \text { The Author(s). Published by S. Karger AG, Basel } \\
\text { www.karger.com/cpb }\end{array}$
\end{tabular} \\ Zhao et al.: Metabolic Alterations in Diabetic Hearts}

10 Burnstock G, Pelleg A: Cardiac purinergic signalling in health and disease. Purinergic Signal 2015;11:1-46.

11 Shockcor JP, Holmes E: Metabonomic applications in toxicity screening and disease diagnosis. Curr Top Med Chem 2002;2:35-51.

12 Nicholson JK: Global systems biology, personalized medicine and molecular epidemiology. Mol Syst Biol 2006;2:52.

13 Nicholson JK, Lindon JC, Holmes E: 'Metabonomics': understanding the metabolic responses of living systems to pathophysiological stimuli via multivariate statistical analysis of biological NMR spectroscopic data. Xenobiotica 1999;29:1181-1189.

14 Psihogios NG, Gazi IF, Elisaf MS, Seferiadis KI, Bairaktari ET: Gender-related and age-related urinalysis of healthy subjects by NMR-based metabonomics. NMR Biomed 2008;21:195-207.

15 Zhao LC, Zhang XD, Wang HY, Lin DH: 1H NMR-Based Metabonomic Analysis of Metabolic Changes of Serum and Liver in Zucker Obese Rats. Analytical Letters 2011;44:1579-1590.

16 Zhao LC, Zhang XD, Liao SX, Gao HC, Wang HY, Lin DH: A metabonomic comparison of urinary changes in Zucker and GK rats. J Biomed Biotechnol 2010;2010:431894.

17 Zhao L, Gao H, Lian F, Liu X, Zhao Y, Lin D: (1)H-NMR-based metabonomic analysis of metabolic profiling in diabetic nephropathy rats induced by streptozotocin. Am J Physiol Renal Physiol 2011;300:F947-956.

18 Wei T, Zhao L, Jia J, Xia H, Du Y, Lin Q Lin X, Ye X, Yan Z, Gao H: Metabonomic analysis of potential biomarkers and drug targets involved in diabetic nephropathy mice. Sci Rep 2015;5:11998.

19 Guan M, Xie L, Diao C, Wang N, Hu W, Zheng Y, Jin L, Yan Z, Gao H: Systemic perturbations of key metabolites in diabetic rats during the evolution of diabetes studied by urine metabonomics. PLoS One 2013;8:e60409.

20 Kilkenny C, Browne WJ, Cuthill IC, Emerson M, Altman DG: Improving bioscience research reporting: the ARRIVE guidelines for reporting animal research. PLoS Biol 2010;8:e1000412.

21 Zhang X, Pan L, Yang K, Fu Y, Liu Y, Chi J, Zhang X, Hong S, Ma X, Yin X: H3 Relaxin Protects Against Myocardial Injury in Experimental Diabetic Cardiomyopathy by Inhibiting Myocardial Apoptosis, Fibrosis and Inflammation. Cell Physiol Biochem 2017;43:1311-1324.

-22 Zhao L, Zhang H, Yang Y, Zheng Y, Dong M, Wang Y, Bai G, Ye X, Yan Z, Gao H: Serum metabonomic analysis of protective effects of Curcuma aromatica oil on renal fibrosis rats. PLoS One 2014; 9:e108678.

-23 Westerhuis JA, van Velzen EJ, Hoefsloot HC, Smilde AK: Multivariate paired data analysis: multilevel PLSDA versus OPLSDA. Metabolomics 2010;6:119-128.

24 Cloarec O, Dumas ME, Trygg J, Craig A, Barton RH, Lindon JC, Nicholson JK, Holmes E: Evaluation of the orthogonal projection on latent structure model limitations caused by chemical shift variability and improved visualization of biomarker changes in $1 \mathrm{H}$ NMR spectroscopic metabonomic studies. Anal Chem 2005;77:517-526.

-25 Zheng H, Yde CC, Clausen MR, Kristensen M, Lorenzen J, Astrup A, Bertram HC: Metabolomics investigation to shed light on cheese as a possible piece in the French paradox puzzle. J Agric Food Chem 2015;63:28302839.

26 Zhang S, Nagana Gowda GA, Asiago V, Shanaiah N, Barbas C, Raftery D: Correlative and quantitative 1H NMR-based metabolomics reveals specific metabolic pathway disturbances in diabetic rats. Anal Biochem 2008;383:76-84.

-27 Liu Y, Yan X, Mao G, Fang L, Zhao B, Liu Y, Tang H, Wang N: Metabonomic profiling revealed an alteration in purine nucleotide metabolism associated with cardiac hypertrophy in rats treated with thiazolidinediones. J Proteome Res 2013;12:5634-5641.

28 Boudina S, Abel ED: Mitochondrial uncoupling: a key contributor to reduced cardiac efficiency in diabetes. Physiology (Bethesda) 2006;21:250-258.

29 Zhou Y, Wu W: The Sodium-Glucose Co-Transporter 2 Inhibitor, Empagliflozin, Protects against Diabetic Cardiomyopathy by Inhibition of the Endoplasmic Reticulum Stress Pathway. Cell Physiol Biochem 2017;41:2503-2512.

-30 Huang Z, Zhuang X, Xie C, Hu X, Dong X, Guo Y, Li S, Liao X: Exogenous Hydrogen Sulfide Attenuates High Glucose-Induced Cardiotoxicity by Inhibiting NLRP3 Inflammasome Activation by Suppressing TLR4/NFkappaB Pathway in H9c2 Cells. Cell Physiol Biochem 2016;40:1578-1590.

-31 Giannini S, Serio M, Galli A: Pleiotropic effects of thiazolidinediones: taking a look beyond antidiabetic activity. J Endocrinol Invest 2004;27:982-991. 


\section{Cellular Physiology Cell Physiol Biochem 2018;48:934-946 \begin{tabular}{l|l} 
DOI: 10.1159/000491960 & $\begin{array}{l}\text { O } 2018 \text { The Author(s). Published by S. Karger AG, Basel } \\
\text { www.karger.com/cpb }\end{array}$
\end{tabular} \\ Zhao et al.: Metabolic Alterations in Diabetic Hearts}

-32 Javadov S, Jang S, Agostini B: Crosstalk between mitogen-activated protein kinases and mitochondria in cardiac diseases: therapeutic perspectives. Pharmacol Ther 2014;144:202-225.

-33 Boudina S, Sena S, Theobald H, Sheng X, Wright JJ, Hu XX, Aziz S, Johnson JI, Bugger H, Zaha VG, Abel ED: Mitochondrial energetics in the heart in obesity-related diabetes: direct evidence for increased uncoupled respiration and activation of uncoupling proteins. Diabetes 2007;56:2457-2466.

-34 Baseler WA, Dabkowski ER, Williamson CL, Croston TL, Thapa D, Powell MJ, Razunguzwa TT, Hollander JM: Proteomic alterations of distinct mitochondrial subpopulations in the type 1 diabetic heart: contribution of protein import dysfunction. Am J Physiol Regul Integr Comp Physiol 2011;300:R186-200.

-35 Riddle MC: Effects of Intensive Glucose Lowering in the Management of Patients With Type 2 Diabetes Mellitus in the Action to Control Cardiovascular Risk in Diabetes (ACCORD) Trial. Circulation 2010;122:844-846.

-36 Reusch JEB, Wang CCL: Cardiovascular Disease in Diabetes: Where Does Glucose Fit In? J Clin Endocr Metab 2011;96:2367-2376.

37 Lopaschuk GD, Ussher JR, Folmes CDL, Jaswal JS, Stanley WC: Myocardial Fatty Acid Metabolism in Health and Disease. Physiol Rev 2010;90:207-258.

38 Taegtmeyer H, McNulty P, Young ME: Adaptation and maladaptation of the heart in diabetes: Part I General concepts. Circulation 2002;105:1727-1733.

-39 Avogaro A, Vigili de Kreutzenberg S, Negut C, Tiengo A, Scognamiglio R: Diabetic cardiomyopathy: a metabolic perspective. Am J Cardiol 2004;93:13A-16A.

$>40$ Sas KM, Karnovsky A, Michailidis G, Pennathur S: Metabolomics and Diabetes: Analytical and Computational Approaches. Diabetes 2015;64:718-732.

41 Neishabouri SH, Hutson SM, Davoodi J: Chronic activation of mTOR complex 1 by branched chain amino acids and organ hypertrophy. Amino Acids 2015;47:1167-1182.

-42 Blomstrand E, Eliasson J, Karlsson HKR, Kohnke R: Branched-chain amino acids activate key enzymes in protein synthesis after physical exercise. J Nutr 2006;136:269-273.

43 Yang F, Zhang L, Gao Z, Sun X, Yu M, Dong S, Wu J, Zhao Y, Xu C, Zhang W, Lu F: Exogenous H2S Protects Against Diabetic Cardiomyopathy by Activating Autophagy via the AMPK/mTOR Pathway. Cell Physiol Biochem 2017;43:1168-1187.

44 O'Connell T: The Complex Role of Branched Chain Amino Acids in Diabetes and Cancer. Metabolites 2013;3:931-945.

45 Bak LK, Waagepetersen HS, Sorensen M, Ott P, Vilstrup H, Keiding S, Schousboe A: Role of branched chain amino acids in cerebral ammonia homeostasis related to hepatic encephalopathy. Metabolic Brain Disease 2013;28:209-215.

46 Li T, Zhang Z, Kolwicz SC, Jr., Abell L, Roe ND, Kim M, Zhou B, Cao Y, Ritterhoff J, Gu H, Raftery D, Sun H, Tian R: Defective Branched-Chain Amino Acid Catabolism Disrupts Glucose Metabolism and Sensitizes the Heart to Ischemia-Reperfusion Injury. Cell Metab 2017;25:374-385. 